TITLE:

\title{
Mixing speed-controlled gold nanoparticle synthesis with pulsed mixing microfluidic system
}

\section{AUTHOR(S):}

Sugano, Koji; Uchida, Yuki; Ichihashi, Osamu; Yamada, Hideo; Tsuchiya, Toshiyuki; Tabata, Osamu

\section{CITATION:}

Sugano, Koji ... [et al]. Mixing speed-controlled gold nanoparticle synthesis with pulsed mixing microfluidic system. Microfluidics and Nanofluidics 2010, 9(6): 1165-1174

\section{ISSUE DATE:}

2010-12

URL:

http://hdl.handle.net/2433/134601

\section{RIGHT:}

The final publication is available at www.springerlink.com; This is not the published version. Please cite only the published version.; この論文 は出版社版でありません。引用の際には出版社版をご確認ご利用くだ さい。 


\title{
Mixing Speed-Controlled Gold Nanoparticle Synthesis with Pulsed Mixing Microfluidic System
}

Koji Sugano*, Yuki Uchida, Osamu Ichihashi, Hideo Yamada, Toshiyuki Tsuchiya and Osamu Tabata

Department of Micro Engineering, Graduate School of Engineering, Kyoto University, Yoshida-Honmachi, Sakyo-ku, Kyoto 606-8501, Japan

Tel: $81-75-753-5250$

Fax: 81-75-753-5250

Email: sugano@me.kyoto-u.ac.jp

\begin{abstract}
Gold nanoparticles with diameters of a few tens of nanometer and a narrow size distribution were synthesized using a pulsed mixing method with a microfluidic system which consists of a Y-shaped mixing microchannel and two piezoelectric valveless micropumps. This mixing method enables control of the mixing speed of gold salts and reducing agent by changing the switching frequency of the micropumps, which was our focus to improve the particle size distribution, which is an essential parameter in gold nanoparticle synthesis. In the proposed method, the mixing time was inversely proportional to the switching frequency and the minimum mixing time was $95 \mathrm{msec}$ at a switching frequency of $200 \mathrm{~Hz}$. During synthesis experiments, the mean diameter of the synthesized gold nanoparticles was found to increase and the coefficient of variation of particle size was found to decrease with decreasing mixing time. We successfully improved the coefficient of variation to less than $10 \%$ for a mean diameter of around $40 \mathrm{~nm}$.
\end{abstract}

Keywords: Gold nanoparticle, Mixing speed, Pulsed mixing, Microfluidic device, Micropump

\section{Introduction}

Gold nanoparticles have attracted significant scientific and commercial interest as nano materials since their chemical and physical properties are different from those of bulk materials due to quantum size effects [Henglein 1993], high reactivity [Nakamura et al. 2004] and localized surface plasmons (LSPs) [Nath et al. 2002]. The presence of LSPs leads to a strong enhancement of the electric field around a gold nanoparticle when incident light in the visible region interacts with a collective oscillation of electrons in the nanoparticle, and therefore it has attracted the attention of chemists since an electric field enhancement was found to be crucial for highsensitivity bio/chemical analysis using Surface Enhanced Raman Spectroscopy (SERS) [Shibamoto et al. 2001]. Furthermore, in recent years, many applications of LSPs have emerged in the nanophotonic field [Maier et al. 2003, Grigorenko et al. 2005].

In such applications, both the average size of the gold nanoparticles and the size distribution are critically important factors influencing LSP properties due to the strong dependence of these properties on particle size [Ekardt 1989]. For example, gold nanoparticles with diameters of 60 $\mathrm{nm}$ are known to produce the largest enhancement of the electric field, making them highly 
suitable for application to SERS [Quinten and Kreibig 1986]. Therefore, there has been much interest in developing a particle fabrication process that allows good controllability of the particle size and the size distribution.

As fabrication methods for colloidal gold nanoparticles, laser ablation [Mafune et al. 2001] and various liquid-phase synthesis methods by reduction of gold salts have been reported. In almost all cases, the latter approach has been taken and various reduction methods such as ultrasonic [Nagata et al. 1996], ultraviolet [Esumi et al. 1998] and chemical methods [Jana et al. 2001, Turkevich et al. 1951, Frens 1973, Cow and Zukoski 1994, Freund and Spiro 1982, Muhlpfordt 1982, Chiang 2000, Nakamoto et al. 2005] have been reported. The chemical reduction method with a reducing agent has been most frequently used for scientific studies and commercial production since it allows the synthesis of large quantities of nanoparticles and offers better control of the mean particle size by changing the type of reducing agent, reaction temperature, and concentration of the solution. However, the size distribution becomes wider with increasing particle diameter and achieving a narrow distribution for a mean diameter of more than a few tens of nm has proven difficult [Jana et al. 2001, Turkevich et al. 1951, Frens 1973, Cow and Zukoski 1994, Freund and Spiro 1982]. We considered a possible approach to address this difficulty based on a previously proposed formation mechanism for gold nanoparticles [Sugimoto 2003].

In the liquid-phase chemical reduction method, gold nanoparticles are generated by aggregation of gold atoms resulting from the reduction of gold salt by a reducing agent. As shown in Fig. 1, this process is divided into two stages: the nucleation and nucleus-growth stage (Stage 1), and the nucleus-growth-only stage (Stage 2), depending on the concentration of gold atoms in the mixed solution of gold salt and reducing agent. In the early stages of mixing, the gold atom concentration increases as the gold salt is chemically reduced. When it increases above a critical supersaturation level, nuclei are produced as aggregated clusters of gold atoms (nucleation) and these grow by further aggregation of gold atoms (nucleus growth). If the gold atom concentration decreases below the critical supersaturation level due to consumption of gold atoms by nucleation and growth, the nucleation process ceases and nucleus growth becomes dominant.

The size of the nanoparticles produced depends on (a) the number and size of nuclei generated per unit volume of solution during Stage 1, and (b) the number of remaining gold atoms per unit volume during Stage 2. Smaller particles are produced when a large number of nuclei are generated in Stage 1 since only a small amount of gold atoms is available for their further growth. 
The number of generated nuclei depends on their generation speed (namely, chemical reaction speed), the concentration of the solution and the duration of the nucleation process.

The size distribution of the nanoparticles is dominated mainly by the size uniformity of the clusters generated during Stage 1. A longer nucleation process gives rise to a larger size distribution since additional nuclei are being continuously formed.

Based on this particle synthesis and growth mechanism, many researchers have tried to control the number of generated nuclei and the size uniformity of clusters during Stage 1 by varying factors related to the chemical reaction such as solution concentration, reaction temperature and the type of reducing agent. Sodium citrate is one of the most well-known reducing agents for gold nanoparticles more than a few tens of $\mathrm{nm}$ in diameter. The synthesis method reported by Frens allows for control of the gold nanoparticle diameter from about 10 to $150 \mathrm{~nm}$. However, as mentioned previously, it has so far proven difficult to obtain a narrow size distribution for nanoparticles more than a few tens of $\mathrm{nm}$ in diameter. We believe that this problem arises with conventional methods as a result of nonuniform reaction speed due to temperature inhomogeneity and long mixing times which give rise to a long nucleation period during Stage 1. The nonunifrom reaction speed at different places in a reaction chamber during an early stage of reaction leads different reaction routes of nucleation and nuclei growth with time lag as shown in Fig. 1. This difference leads the long nucleation period which results in wide distribution of particle size.

A microfluidic system is a promising candidate for nanoparticle synthesis because highly uniform reaction temperatures and solution concentrations can be achieved in the confined micronscale reaction chamber, and several studies have been reported on the synthesis of metal nanoparticles using such microfluidic systems. In most cases, the target particle diameter was less than $10 \mathrm{~nm}$ [Wagner et al. 2008, Lin et al. 2004, Shalom et al. 2007, deMello et al. 2004]. Furthermore, some researchers attempted to produce specific particle shapes such as rods and plates [Boleininger et al. 2006, Weng et al.]. However, there have been few reports on the particle size distribution in microfluidic systems. The goal of the present study is to synthesize spherical nanoparticles with diameters of a few tens of nm with a narrow size distribution. Wagner et al. reported a mean particle diameter of approximately $30 \mathrm{~nm}$ with a coefficient of variation of about $30 \%$ [Wagner and Kohler 2005]. They used laminar flow mixing in a microchannel a few hundred microns in width, with a rather low mixing time compared to the 
batch process since mixing occurs by molecular diffusion across the interface between two solutions. We assume that the high coefficient of variation was the result of a low mixing speed under conventional laminar flow conditions, which gives rise to a nonuniform concentration during the early stages of mixing. This means the different routes as shown in Fig. 1 still occur even using the microfluidic devices. Therefore, the mixing time should be reduced in order to decrease the coefficient of variation. The time lag as shown in Fig. 1 is expected to be reduced by short mixing time. Yang et al. reported a new microfluidic device for synthesis of particles with sizes ranging from 19 to $58 \mathrm{~nm}$ using rapid mixing during a period of about 1 second [Yang et al. 2010]. However, no evaluation results of the size distribution were reported in that paper.

To date, there have been no reports on the influence of mixing time on the particle size distribution. Therefore, in the present study, we examined the effect of mixing time on the coefficient of variation for mixing times of less than 1 second. To control the mixing time, a pulsed mixing method [Fujii et al. 2003] was adopted in the microfluidic system in the present study. This mixing method enables us to mix two solutions very rapidly and to easily tune the mixing speed. In the present paper, we report on the fabrication and evaluation of a pulsed mixing microfluidic system for gold nanoparticle synthesis and the influence of the mixing time on the mean particle size and size distribution.

\section{Pulsed mixing method}

\subsection{Principle of pulsed mixing method and microfluidic system}

A pulsed mixing method is an efficient way for mixing two solutions and it enables us to shorten and control the mixing time. The microfluidic system used for the pulsed mixing consists of a Yshaped microchannel connected to an expanded microchannel and two piezoelectric valveless micropumps, as shown in Figs. 2 and 3. Figure 3 shows a schematic image of the pulsed mixing method. The height of all sections of the microchannel is $160 \mu \mathrm{m}$. The widths of the Y-shaped microchannel at the point of merger and the downstream expanded microchannel are 30 and 150 $\mu \mathrm{m}$, respectively. During pulsed mixing, two solutions are alternately pumped into the Y-shaped microchannel to produce pulsed layers of each solution. These pulsed layers are then introduced into the expanded microchannel so that their thickness decreases. The two solutions subsequently interdiffuse at their common interfaces in the expanded microchannel. The decrease in layer thickness that occurs in the expanded microchannel gives rise to more rapid 
mixing since the diffusion time necessary to mix the two solutions is proportional to the square of the layer thickness. The layer thickness is determined by the flow rate and the switching frequency of the two solutions. The two valveless micropumps in the microfluidic system can precisely control flow parameters such as flow rate and switching response for the pulsed mixing method.

This mixing method can be expected to tune the mixing speed by only changing the switching frequency of two solutions. Therefore this method is more suitable than other passive micromixer, for example, split-and-recombine micromixer which achieved significantly rapid mixing decreasing diffusion length of two solutions by stacking of fluid lamellas [Bessoth et al. 1999].

\subsection{Piezoelectric valveless micropump}

We used the micropump which working principle is dependency of flow resistances of inlet and outlet channels on flow rate for the pulsed mixing on the model of the previous work [Fujii et al. 2003] although some valveless micropumps have been reported [Gerlach 1998, Gerlach et al. 1995]. Figure 4 shows a schematic cross-sectional view and a top view of the piezoelectric valveless micropump. The micropump consists of an inlet channel $(25-\mu \mathrm{m}$ width, $25-\mu \mathrm{m}$ height and $25-\mu \mathrm{m}$ length), a pair of outlet channels (36- $\mu \mathrm{m}$ width, $25-\mu \mathrm{m}$ height and $440-\mu \mathrm{m}$ length for each channel) and a pump chamber with a diaphragm (30- $\mu \mathrm{m}$ thickness). A piezoelectriczirconate-titanate (PZT) plate with a thickness of $60 \mu \mathrm{m}$ is located on the backside of the diaphragm. The structure of this micropump and the driving pulse were designed by equivalent electrical circuit analysis [Hayamizu et al. 2002, Tanaka et al. 2007].

\section{Experimental}

\subsection{Fabrication process of microfluidic system}

The microfluidic system was fabricated by silicon ( $\mathrm{Si}$ ) micromachining as shown in Fig. 5 . Silicon dioxide $\left(\mathrm{SiO}_{2}\right)$ with a thickness of $1 \mu \mathrm{m}$ is formed on a $200-\mu \mathrm{m}$-thick $\mathrm{Si}$ wafer by thermal oxidation. Following deposition of a $0.1-\mu$ m-thick aluminum (Al) layer, the $\mathrm{Al}$ and $\mathrm{SiO}_{2}$ layers are patterned $(\mathrm{a}, \mathrm{b})$. Additional patterning is then carried out on the Al layer alone to produce a stepped Si structure (c). The backside $\mathrm{SiO}_{2}$ layer is also patterned (d). The Si substrate is then etched by Reactive Ion Etching (RIE) using the $\mathrm{SiO}_{2}$ and $\mathrm{Al}$ as an etching mask (e). The $\mathrm{Si}$ 
backside is also etched to allow alignment of the PZT plate with the Si diaphragm (f). After removal of the topside $\mathrm{SiO}_{2}$ regions uncovered by $\mathrm{Al}$, the $\mathrm{Si}$ is etched by $\mathrm{RIE}$ using the $\mathrm{Al}$ as a mask pattern $(\mathrm{g}, \mathrm{h})$. An oxide layer of $1 \mu \mathrm{m}$ is then formed on the Si surface to produce a hydrophilic surface (i). $\mathrm{Cr} / \mathrm{Au}(10 \mathrm{~nm} / 100 \mathrm{~nm})$ layers are next deposited on the wafer backside for the PZT electrode (j). Pyrex glass with thickness of $700 \mu \mathrm{m}$ is then bonded to the Si structure by anodic bonding $(\mathrm{k})$. Finally, the PZT plate with thickness of $50 \mu \mathrm{m}$ is glued onto the etched backside groove (1).

\subsection{Pulsed mixing}

The performance of the fabricated micropump was characterized by its flow rate and output pressure. A glass tube was connected to the outlet hole of the microchannel and the displacement of the water surface was measured. The flow rates of the two micropumps on the chip were calibrated by adjusting the amplitude of the driving pulse in order to generate identical flow rates from the two micropumps during the experiments. A voltage, ranging from 50 to $100 \mathrm{~V}$, was applied to the micropumps to produce flow rates of $40 \mathrm{nl} / \mathrm{sec}$.

In order to verify the formation of pulsed layers, the pulsed flow was observed with a fluorescence confocal microscope (IX71, Olympus). The fluorescent dye used in this experiment was Rhodamine B. Fluorescence images (16-bit grayscale) were recorded by a CCD camera at mid-depth in the microchannel. One micropump was filled with the Rhodamin B which was diluted with ultrapure water to a concentration of $48 \mathrm{mg} / \mathrm{L}$, and the other was filled with ultrapure water alone. The fluorescence intensity was confirmed to be proportional to the concentration of fluorescent dye for concentrations less than $48 \mathrm{mg} / \mathrm{L}$. As mixing of the fluorescent dye and ultrapure water proceeded by pulsed mixing in the expanded microchannel, the fluorescence intensity in the microchannel approached half the maximum value observed in the channel filled with only Rhodamine B. In this study, the thickness of the pulsed layer is defined along the center of the microchannel as shown in Fig. 3.

\subsection{Synthesis of gold nanoparticles}

Tetrachloroauric acid $\left(\mathrm{HAuCl}_{4}\right.$, Wako) as a gold salt and sodium citrate $\left(\mathrm{Na}_{3} \mathrm{C}_{6} \mathrm{H}_{5} \mathrm{O}_{7}\right.$, Kanto chemicals) as a reducing agent were mixed at room temperature. Table 1 shows the experimental conditions for the gold salt $(0.48$ or $0.96 \mathrm{mM})$, reducing agent $(1.44$ or $2.88 \mathrm{mM})$, mixing method and collection method of the mixed solution. 
In the first experiments (collection method 1), the mixed solution was allowed to accumulate on the glass at the outlet port of the microchannel (see Fig. 2) for 5 minutes and was then collected into a test-tube by a pipette. In the second experiment (collection method 2), the mixed solution in the microchannel was discharged for 5 minutes into the silicone tube with an inner diameter of $500 \mu \mathrm{m}$, which was connected to the outlet port. The collected solutions by both collection 1 and 2 were kept under dark storage for 2 days to assure complete growth. They were then dropped onto a Si chip whose surface was modified with 3-Aminopropyltriethoxy-silane (Sigma Aldrich) to fix gold nanoparticles.

Diameters of synthesized gold nanoparticles were measured by Scanning Electron Microscopy (SEM; S-4500, Hitachi). For each solution, the number of particles counted was 150 . Gold nanoparticles synthesized by a conventional batch process were also prepared for comparison.

\section{Results and discussion}

\subsection{Pulsed mixing}

Figure 6 shows fluorescence microscope images of pulsed layer formation at switching frequencies of (a) $50 \mathrm{~Hz}$, (b) $100 \mathrm{~Hz}$ and (c) $200 \mathrm{~Hz}$. As can be seen, the thicknesses of the formed layers decreased as they moved from the Y-shaped microchannel into the expanded microchannel. Pulsed mixing was confirmed at switching frequencies of up to $200 \mathrm{~Hz}$. At higher frequencies, the volume ejected from each pump was insufficient to occupy the entire microchannel width upon emerging from the Y-shaped microchannel and the flow pattern of the two solutions resembled that during laminar flow mixing. From the images in Fig. 6, the variation in the fluorescence intensity as a function of position along the Y-shaped and expanded microchannels was calculated as shown in Fig. 7. Clear cyclical variations in the fluorescence intensity can be observed along the microchannel. Furthermore, the intensity gradually approaches half of its maximum value, which indicates that mixing of two solutions is finished.

From the cycle length of the variations in fluorescent intensity in Fig. 7, the thicknesses of pulsed layers in the Y-shaped and expanded microchannel were measured (Fig. 8). The measured thicknesses in the Y-shaped microchannel were 269,141 and $72 \mu \mathrm{m}$, for frequencies of 50, 100 and $200 \mathrm{~Hz}$, respectively, and the thicknesses in the expanded microchannel were 78, 41 and $21 \mu \mathrm{m}$, respectively. The thicknesses of the pulsed layers were inversely proportional to the switching frequency in both the Y-shaped and expanded microchannels. 
The volume required to fill the dotted circle in Fig. 3 is $7.1 \times 10^{4} \mu \mathrm{m}^{3}$, which is the necessary volume to form a pulsed layer. The ejected volume of $2.0 \times 10^{5} \mu \mathrm{m}^{3}$ at the switching frequency of $200 \mathrm{~Hz}$ is therefore sufficient to form a pulsed layer.

In addition, the pulsed layer thicknesses along the center of the microchannel were calculated based on the flow velocity and the switching frequency. The thickness of a pulsed layer $w$ can be expressed as $w=U_{\max } / f$, where $U_{\max }$ and $f$ indicate the maximum flow velocity in the channel cross section and the switching frequency, respectively. $U_{\max }$ has a value of $1.5 U$ and $2.1 U$ in the 30- $\mu \mathrm{m}$-wide (aspect ratio: 5) and 150- $\mu \mathrm{m}$-wide (aspect ration: 1) channels, respectively, where $U$ represents the average velocity in the channel. The calculated layer thicknesses are also plotted in Fig. 8, and it can be seen that the experimental and calculated values show good agreement.

Figure 9 shows the mixing time as a function of the switching frequency. The mixing time is defined as the time until the difference between the measured fluorescence intensity and half the maximum intensity becomes less than $5 \%$. The mixing times were found to be 448, 232 and 95 msec for switching frequencies of 50, 100 and $200 \mathrm{~Hz}$, respectively. Therefore, the mixing time can be easily controlled by changing the switching frequency and rapid mixing was achieved by increasing the switching frequency up to $200 \mathrm{~Hz}$ in this study.

The theoretical mixing time $t$ was also calculated as $t=l^{2} / 2 D$, where $l$ and $D$ indicate the diffusion length and the diffusion constant, respectively. A diffusion constant of $4.27 \times 10^{-6}$ $\mathrm{cm}^{2} / \mathrm{sec}$ [Culbertson et al. 2002] was used in this calculation. The diffusion length $l$ was defined as distance from Point A to the nearest interface between two solutions since the slowest mixing point is Point A in Fig. 3. The calculated results are also shown in Fig. 9, and agree well with the experimental data. These results confirm that control of the mixing time is possible using the fabricated device.

\subsection{Synthesis of gold nanoparticles}

Gold nanoparticles were produced using a batch process and by pulsed mixing at switching frequencies of 50, 100 and $200 \mathrm{~Hz}$, and SEM images of the nanoparticles are shown in Fig. 10. Figure 11 shows the size distributions of the produced gold nanoparticles measured from the SEM images. The conditions A and B in Figs. 10-12 correspond to collection methods 1 and 2, respectively, where the same concentration of $\mathrm{HAuCl}_{4}$ was used. Figure 12 shows the mean 
particle diameter and the coefficient of variation of particle diameter as a function of the switching frequency.

\subsubsection{Coefficient of variation of particle diameter}

According to Figs. 10-12, as a general trend, the coefficient of variation is smaller for nanoparticles produced by the pulsed mixing method than by the batch process. Furthermore, the coefficient of variation also decreases with increasing switching frequency. These results indicate that the coefficient of variation is smaller for shorter mixing times of less than a few hundred msec. This corresponds well with the proposed formation mechanism of nanoparticles described in the introduction. The results also suggest that the initial reaction between $\mathrm{HAuCl}_{4}$ and sodium citrate, which corresponds to the nucleation process, is a diffusion-limited reaction.

We now discuss the effect of the collection method on the coefficient of variation by comparing the results for the A and B groups in Figs. 10-12. The reason why a switching frequency of 50 $\mathrm{Hz}$ and collection method 1 led to a higher coefficient of variation than that for the batch process is thought to be as follows. In collection method 1, the mixed solution is accumulated at the outlet port for 5 minutes. During this accumulation time, the particles which experienced different synthesis times are mixed at the outlet port. Therefore this accumulated solution includes the different particle size. These particles grow with gold atoms which are also mixed up at the outlet port. This influence increases the coefficient of variation. This effect imposes the same effect as the low-mixing-speed effect to the particle synthesis. The low mixing speed leads time lag of the nucleation and nucleus growth due to the diffusion-limited reaction as shown in Fig. 1. This means the solution mixed with the low mixing speed includes particles which experienced different synthesis times.

At switching frequencies of 100 and $200 \mathrm{~Hz}$, the coefficients of variation using collection method 1 (Figs. 10-12 A) were smaller than those of collection method 2 (Figs. 10-12 B). However, for synthesis by chemical reduction, the coefficient of variation increases with increasing particle diameter. Considering that the mean particle diameters for collection method 1 were much smaller than those for collection method 2, it can be said that collection method 2 produces a larger relative improvement in the coefficient of variation. In other word, during the synthesis, solutions which have experienced different reaction times should not become mixed as the collection method 2 .

We now consider the effect of mixing time on the coefficient of variation in the case of 
collection method 2. The coefficient of variation was smaller for a switching frequency of 100 $\mathrm{Hz}$ than for $50 \mathrm{~Hz}$. However, the coefficients of variation at switching frequencies of 100 and $200 \mathrm{~Hz}$ were almost the same. When the mixing speed is changed, the nucleation period is the only factor influencing the coefficient of variation. The almost identical coefficients of variation at 100 and $200 \mathrm{~Hz}$ possibly indicate that the mixing time at $100 \mathrm{~Hz}$ was short enough compared to the nucleation period to mix the two solutions uniformly.

Concerning the dependence of the coefficient of variation on the $\mathrm{HAuCl}_{4}$ concentration, it was found that a higher concentration of the gold salt led to a lower coefficient of variation. The coefficient variation for a $\mathrm{HAuCl}_{4}$ concentration of $0.96 \mathrm{mM}$ was much lower than that for a concentration of $0.48 \mathrm{mM}$. A higher concentration of the gold salt not only increases the reduction rate of the salt itself but also increases the consumption rate of gold atoms. Therefore, the nucleation period should be shorter at higher salt concentrations, which would likely lead to a smaller coefficient of variation. A minimum coefficient of variation of $9.6 \%$ was achieved for a $\mathrm{HAuCl}_{4}$ concentration of $0.96 \mathrm{mM}$ and switching frequencies of 100 or $200 \mathrm{~Hz}$. This is the smallest value ever reported for a mean particle diameter of around $40 \mathrm{~nm}$.

\subsubsection{Particle diameter}

The mean diameter of gold nanoparticles produced by pulsed mixing was much larger than that for the batch process except for the case of using collection method $1 . \mathrm{A} \mathrm{HAuCl}_{4}$ concentration of $0.48 \mathrm{mM}$ resulted in a maximum particle diameter of $42.7 \mathrm{~nm}$ at a switching frequency of 200 $\mathrm{Hz}$ using collection method 2. The mean particle diameter increased with increasing switching frequency. These particle diameters are about twice those for the batch process or using collection method 1. These results indicate that a higher mixing speed produces larger particles in the same concentration. This means that the mixing speed affects the number of nuclei produced, which determines the particle size as discussed in the introduction.

\section{Conclusion}

Gold nanoparticles were synthesized using a pulsed mixing method with a microfluidic system in order to achieve a narrow size distribution. In this study, we successfully demonstrated that the pulsed mixing method decreases the coefficient of variation of particle diameter due to rapid mixing.

The fabricated microfluidic system consists of a Y-shaped microchannel and two piezoelectric 
valveless micropumps to allow high-frequency switching during pulsed flow. Pulsed flow was confirmed up to a switching frequency of $200 \mathrm{~Hz}$. It was shown that the mixing time could be easily controlled by changing the switching frequency and rapid mixing was possible by increasing the switching frequency. The mixing time at $200 \mathrm{~Hz}$ was $95 \mathrm{msec}$.

The synthesis experiments established the effect of mixing speed on mean particle size and the size distribution for the first time. The size of the gold nanoparticles was found to increase and the coefficient of variation found to decrease by increasing the mixing speed using the pulsed mixing method. The minimum coefficient of variation was $9.6 \%$ for a $\mathrm{HAuCl}_{4}$ concentration of $0.98 \mathrm{mM}$ using collection method 2. Based on these results, we confirmed that high mixing speed was a critical factor for producing a narrow size distribution because of the short nucleation time involved.

\section{Acknowledgment}

This work was conducted in Kyoto-Advanced Nanotechnology Network, and supported by the Ministry of Education, Culture, Sports, Science and Technology (MEXT), Japan. This work was also supported by Grant-in-Aid for Young Scientists B (No.18710078). The authors are grateful to Sadamu Kinoshita for the SEM observations.

\section{References}

Bessoth F G, deMello A J, Manz A (1999) Microstructure for efficient continuous flow mixing. Anal. Commun. 36: 213-15.

Boleininger J, Kurz A, Reuss V and Sonnichsen C (2006) Microfluidic continuous flow synthesis of rod-shaped gold and silver nanocrystals. Physical Chemistry Chemical Physics 8: 3824-3827.

Chiang C L (2000) Controlled Growth of Gold Nanoparticles in Aerosol-OT/Sorbitan Monooleate/Isooctane Mixed Reverse Micelles. Journal of Colloid and Interface Science 230:60 66.

Chow M and ZukoskiC (1994) Gold Sol Formation Mechanism: Role of Colloidal Stability. Journal of Colloid and Interface Science 165:97-109.

Culbertson C T, Jacobson S C, Ramsey J M (2002) Diffusion coefficient measurements in microfluidic devices. Talanta, 56:365-373.

deMello J and deMello A (2004) Microscale reactors: nanoscale products. Lab on a chip 4: 11-15.

Ekardt W (1989) Size-dependent photoabsorption and photoemission of small metal particles. Physical Review B 31.6360-70.

Esumi K, Suzuki A, Aihara N, Usui K, Torigoe K (1998) Preparetion of Gold Colloids with UV Irradiation Using Dendrimers as Stabilizer. Langmuir 14:3157-3159

Fujii T, Sando Y, Higashino K and Fujii Y (2003) A Plug and Play Microfluidic Device. Lab on a Chip 3:193-7.

Frens G (1973) Controlled Nucleation for the Regulation of the Particle Size in Monodisperse 
Gold Suspensions. Nature Physical Science 241:20-22.

Freund P and Spiro M (1985) Colloidal Catalysis: The Effect of Sol Size and Concentration. The journal of Physical Chemistry 89:1074-7.

Gerlach T, Wurmus H (1995) Working principle and performance of the dynamic micropump. Sensors and Actuators A. 50: 135-40.

Gerlach T (1998) Microdiffusers as dynamic passive valves for micropump applications. Sensors and Actuators A 69: 181-91.

Grigorenko1 A, Geim1 A, Gleeson1 H, Zhang1 Y, Firsov A, Khrushchev I and Petrovic J (2005) Nanofabricated media with negative permeability at visible frequencies. Nature 438:17-20

Hayamizu S, Higashino K, Fujii Y, Sando Y and Yamamoto K (2002) New Bi-directional Valveless Silicon Micro Pump Controlled by Driving Waveform. 15th IEEE International Conference on Micro Electro Mechanical Systems (MEMS 2002) 113-6.

Henglein A (1993) Physicochemical Propertied of Small Metal Particles in Solution Microelectrode Reactions, Chemisorption, Composite Metal Particles, and the Atom-to-Metal Transition. The Journal of Physical Chemistry 97:5457-71.

Jana N R, Gearheart L and Murphy C J (2001) Seeding Growth for Size Control of 5-40 nm Diameter Gold Nanoparticles. Langmuir 17:6782-6.

Lin X Z, Terepka A, Yang H (2004) Synthesis of Silver Nanoparticles in a Continuous Flow Tubular Microreactor. NANO LETTERS 4: 2227-2232.

Mafune F, Kohno J, Takeda Y, Kondow T, Sawada H (2001) Formation of Gold Nanoparticles by Laser Ablation in Aqueous Solution of Surfactant. J. Phys. Chem. 105:5114-5120

Maier S, Kik P, Atwater H, Meltzer S, Harel E, Koel B and Requicha A (2003) Local detection of electromagnetic energy transport below the diffraction limit in metal nanoparticle plasmon waveguides. Nature Material 2:229-31.

Muhlpfordt H (1982) The preparation of colloidal particles using tannic acid as an additional agent. Experientia 38:1127-8.

Nakamoto M, Kashiwagi Y and Yamamoto M (2005) Synthesis and size regulation of gold nanoparticles by controlled thermolysis of ammonium gold(I) thiolate in the absence or presence of amines. Inorganica chimica acta 358:4229-36.

Nakamura H, Li X, Wang H, Uehara M, Miyazaki M, Shimizu H and Maeda H (2004) A simple method of self assembled nano-particles deposition on the micro-capillary inner walls and the reactor application for photo-catalytic and enzyme reactions. Chemical Engineering Journal 101:261-8.

Nagata Y, Mizukoshi Y, Okitsu K, Maeda Y (1996) Sonochemical Formation of Gold Particles in Aqueous Solution. Radiation Research 146:333-338.

Nath N and Chilkoti A (2002) A Colorimetric Gold Nanoparticle Sensor to Interrogate Biomolecular Interactions in Real Time on a Surface Analytical Chemistry 74 504-9.

Shibamoto K, Katayama K, Fujinami M and Sawada T (2001) Fundamental Processes of Surface Enhanced Raman Scattering Detected with Transient Reflecting Grating Spectroscopy. Analytical Sciences 17:91-94.

Shalom D, Wootton R, Winkle R, Cottam B, Vilar R, deMello A and Wilde C (2007) Synthesis of thiol functionalized gold nanoparticles using a continuous flow microfluidic reactor. Materials Letters 61:1146-50.

Sugimoto T (2003) Formation of Monodispersed Nano- and Micro-Particles Controlled in Size, 
Shape, and Internal Structure. Chem. Eng. Technol. 26:313-21.

Tanaka S, Ichihashi O, Sugano K, Tsuchiya T and Tabata O (2007) Analysis of Valveless Piezoelectric Micropump Using Electrical Equivalent Circuit Model. The 16th International Conference on Solid-State Sensors, Actuators and Microsystems (Transducers'07) 2183-6.

Turkevich J, Stevenson P and Hillier J (1951) A study of the Nucleation and Growth Processes in the Synthesis of Colloidal Gold. DISCUSSIONS OF THE FARADAY SOCIETY 11:55

Quinten M and Kreibig U (1986) Optical Properties of Aggregates of Small Metal Particles. Surface Science 172:557-77.

Wagner J and Kohler J M (2005) Continuous Synthesis of Gold Nanoparticles in a Microreactor. Nano Letters 5:685-91.

Wagner J, Tshikhudo T R, Kohler J M (2008) Microfluidic generation of metal nanoparticles by borohydride reduction S104-S109.

Weng C-H, Huang C-C, Yeh C-S, Lei H-Y, Lee G-B (2008) Synthesis of hexagonal gold nanoparticles using a microfluidic reaction system. Journal of Micromechanics and Microengineering 18: 035019 (pp8).

Yang S-Y, Cheng F-Y, Yeh C-S, Lee Gwo-bin (2010) Size-controlled synthesis of gold nanoparticles using a micro-mixing system 8: 303-311. 
Fig. 1. Synthesis mechanism of liquid-phase chemical reaction method for gold nanoparticles. Stage (1): nucleation and nucleus growth, Stage (2): nucleus growth. The dotted line indicates a critical supersaturation level which defines a threshold concentration of $\mathrm{Au}^{0}$ atoms for nucleation.

Fig. 2. Photograph of microfluidic system with two micropumps and a Y-shaped microchannel for pulsed mixing.

Fig. 3. Schematic diagram illustrating principle of pulse mixing with two micropumps. Widths of Y-shaped microchannel to produce pulsed layers and downstream expanded microchannel are 30 and $150 \mu \mathrm{m}$, respectively. The white dotted circle at the confluence point indicates the minimum area occupied by the solution for pulsed mixing. Point $\mathrm{A}$ in the expanded channel indicates the center between interfaces of two solutions.

Fig. 4. Schematics of the piezoelectric valveless micropump. a) top view, b) cross-sectional view, c) and d) top views of the inlet and outlet channel, respectively.

Fig. 5. Fabrication process of a microfluidic system with piezoelectric valveless micropumps.

Table 1. Synthesis conditions for gold nanoparticles.

Fig. 6. Fluorescence images of pulse flow at a switching frequency of (a) $50 \mathrm{~Hz}$, (b) $100 \mathrm{~Hz}$ and (c) $200 \mathrm{~Hz}$. Fluorescent dye and ultrapure water emerge from the top and bottom channels, respectively. Widths of the Y-shaped and expanded channels are 30 and $150 \mu \mathrm{m}$, respectively.

Fig. 7. Fluorescence intensity along the center of the microchannel at mid-depth for a switching frequency of a) $50 \mathrm{~Hz}$, b) $100 \mathrm{~Hz}$ and c) $200 \mathrm{~Hz}$. The fluorescence intensity values are relative values.

Fig. 8. Thickness of the pulsed layer as a function of switching frequency on a logarithmic scale. The solid and dotted line show the thickness of layers in the $30-\mu \mathrm{m}$ - and $150-\mu \mathrm{m}$-wide channels, respectively.

Fig. 9. Mixing time as a function of switching frequency.

Fig. 10. SEM images of gold nanoparticles synthesized by batch process and pulsed mixing at switching frequencies of 50, 100 and $200 \mathrm{~Hz}$. Scale bars indicate $200 \mathrm{~nm}$.

Fig. 11. Particle diameter distributions for gold nanoparticles synthesized by the batch process and the pulsed mixing method at switching frequencies of (a) $50 \mathrm{~Hz}$, (b) $100 \mathrm{~Hz}$ and (c) $200 \mathrm{~Hz}$. A) collection method 1 with a $\mathrm{HAuCl}_{4}$ concentration of $0.48 \mathrm{mM}$. B) collection method 2 with a $\mathrm{HAuCl}_{4}$ concentration of $0.48 \mathrm{mM}, \mathrm{C}$ ) collection method 2 with a $\mathrm{HAuCl}_{4}$ concentration of 0.96 $\mathrm{mM}$.

Fig. 12. Mean particle diameter and coefficient of variation for gold nanoparticles synthesized by the batch process and the pulsed mixing method at switching frequencies of (a) $50 \mathrm{~Hz}$, (b) $100 \mathrm{~Hz}$ and (c) $200 \mathrm{~Hz}$. (A) collection method 1 with a $\mathrm{HAuCl}_{4}$ concentration of $0.48 \mathrm{mM}$. (B) collection method 2 with a $\mathrm{HAuCl}_{4}$ concentration of $0.48 \mathrm{mM},(\mathrm{C})$ collection method 2 with a $\mathrm{HAuCl}_{4}$ concentration of $0.96 \mathrm{mM}$. 


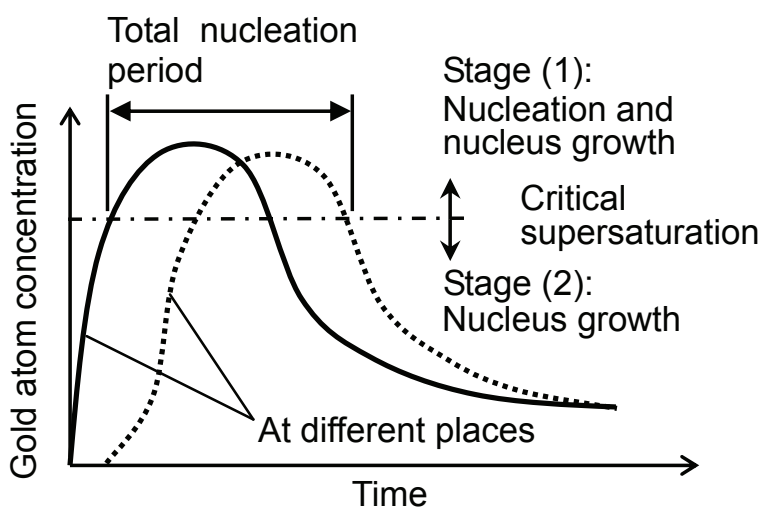

Fig. 1. Synthesis mechanism of liquid-phase chemical reaction method for gold nanoparticles. Stage (1): nucleation and nucleus growth, Stage (2): nucleus growth. The horizontal line indicates a critical supersaturation level which defines a threshold concentration of $\mathrm{Au}^{0}$ atoms for nucleation. The two lines indicate different routes at different places in the reaction chamber, for example at the center and the edge of reaction chamber.

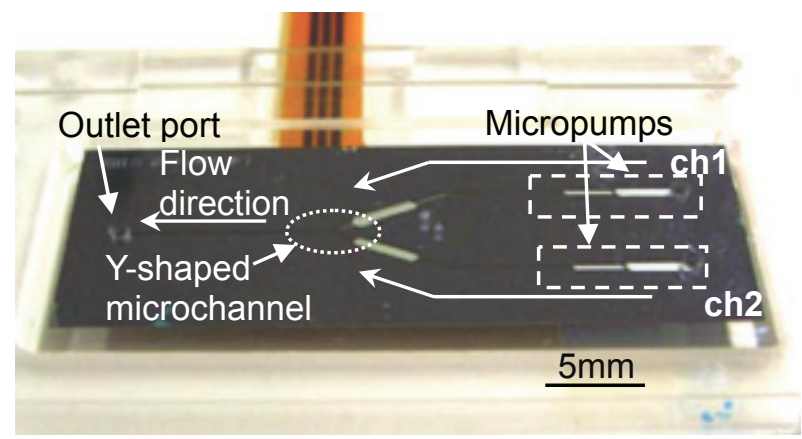

Fig. 2. Photograph of microfluidic system with two micropumps and a Y-shaped microchannel for pulsed mixing.

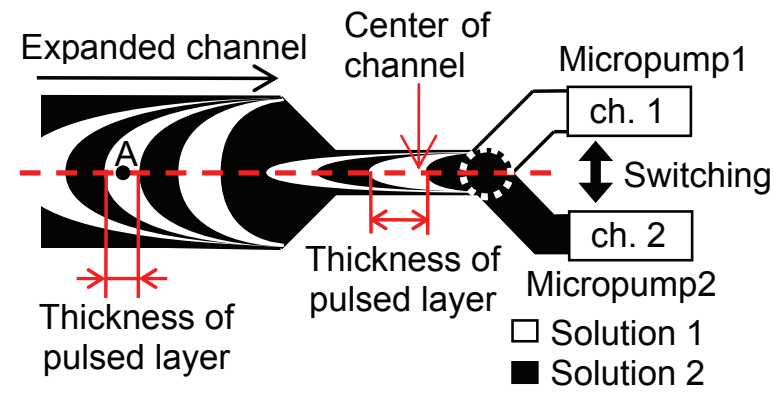

Fig. 3. Schematic diagram illustrating principle of pulse mixing with two micropumps. Widths of Y-shaped microchannel to produce pulsed layers and downstream expanded microchannel are 30 and $150 \mu \mathrm{m}$, respectively. The white dotted circle at the confluence point indicates the minimum area occupied by the solution for pulsed mixing. Point A in the expanded channel indicates the center between interfaces of two solutions. 
a) Top view

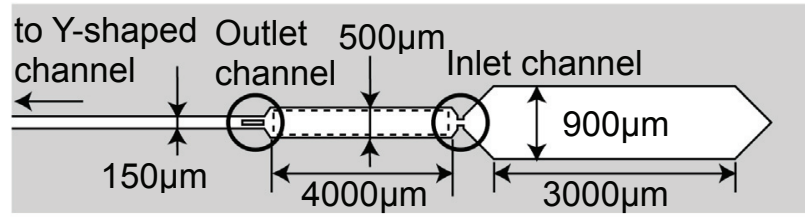

b) Cross-sectional view

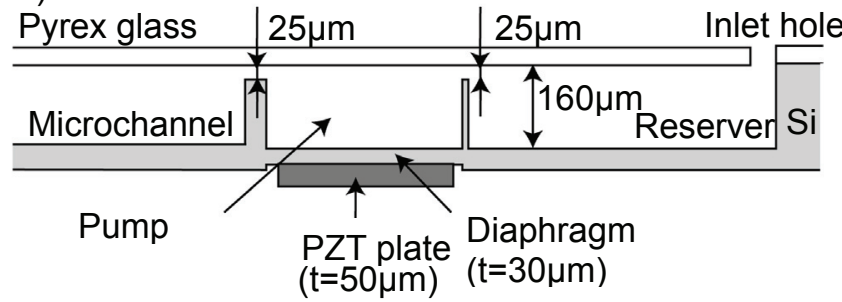

c) Inlet channel

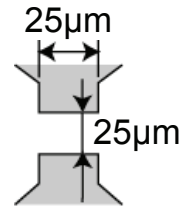

d) Outlet channel

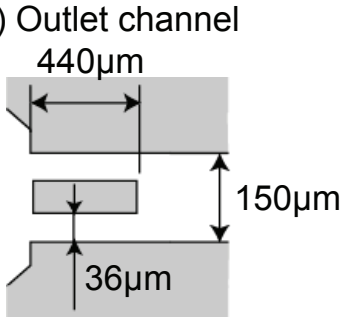

Fig. 4. Schematics of the piezoelectric valveless micropump. a) top view, b) cross-sectional view, c) and d) top views of the inlet and outlet channel, respectively. The schematic is not to scale.

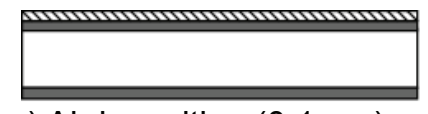

a) Al deposition $(0.1 \mu \mathrm{m})$

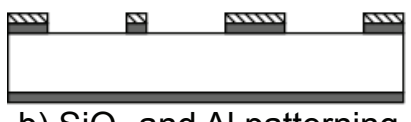

b) $\mathrm{SiO}_{2}$ and $\mathrm{Al}$ patterning

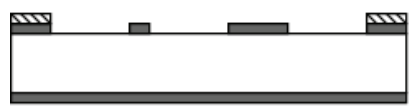

c) Al patterning

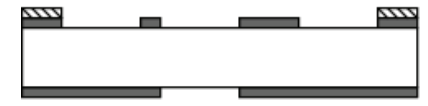

d) Backside $\mathrm{SiO}_{2}$ patterning

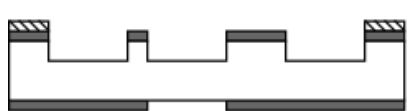

e) 1 st Si etching

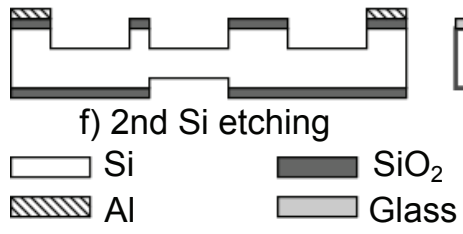

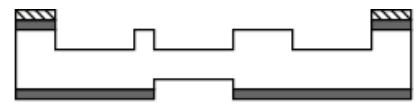

g) $\mathrm{SiO}_{2}$ removal

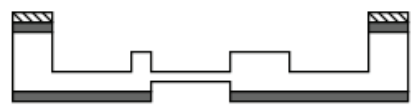

h) 3rd Si etching

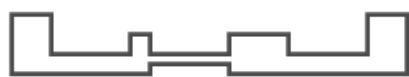

i) Oxidation (1 $\mu \mathrm{m})$

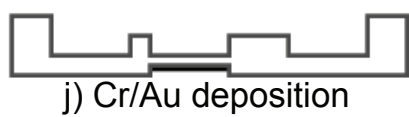

$(10 / 100 \mathrm{~nm})$

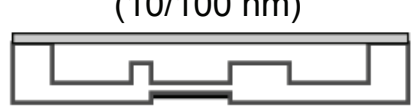

k) Anodic bonding

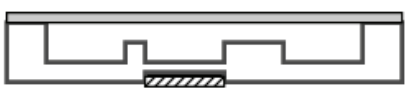

I) PZT bonding

$\mathrm{Cr} / \mathrm{Au}$

चIIIS PZT

Fig. 5. Fabrication process of a microfluidic system with piezoelectric valveless micropumps. 
Table 1. Synthesis conditions for gold nanoparticles.

\begin{tabular}{ccrc}
\hline $\begin{array}{c}\text { Auric acid } \\
{[\mathrm{mM}]}\end{array}$ & $\begin{array}{c}\text { Sodium } \\
\text { citrate [mM] }\end{array}$ & $\begin{array}{c}\text { Switching frequency } \\
{[\mathrm{Hz}] / \text { batch process }}\end{array}$ & $\begin{array}{c}\text { Collection } \\
\text { method }\end{array}$ \\
\hline \hline 0.48 & 1.44 & Batch, 50, 100, 200 & 1 \\
0.48 & 1.44 & $50,100,200$ & 2 \\
0.96 & 2.88 & Batch, 50, 100, 200 & 2 \\
\hline
\end{tabular}

Collection method 1: Accumulated at an outlet port of a microchannel. 2: Discharged and collected into a silicone tube.

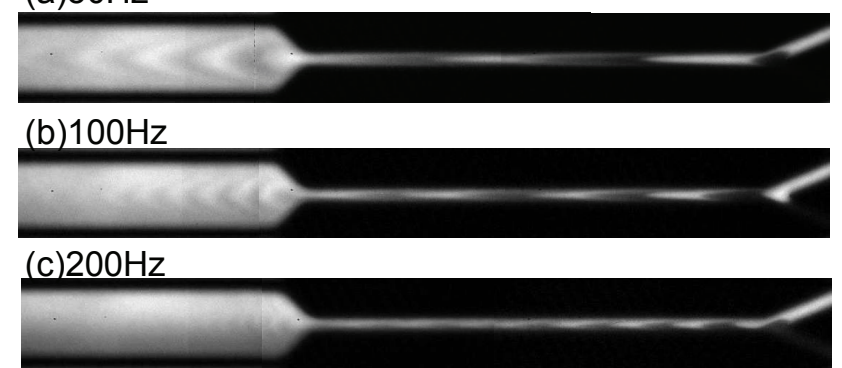

Fig. 6. Fluorescence images of pulse flow at a switching frequency of (a) $50 \mathrm{~Hz}$, (b) $100 \mathrm{~Hz}$ and (c) $200 \mathrm{~Hz}$. Fluorescent dye and ultrapure water emerge from the top and bottom channels, respectively. Widths of the Y-shaped and expanded channels are 30 and $150 \mu \mathrm{m}$, respectively.

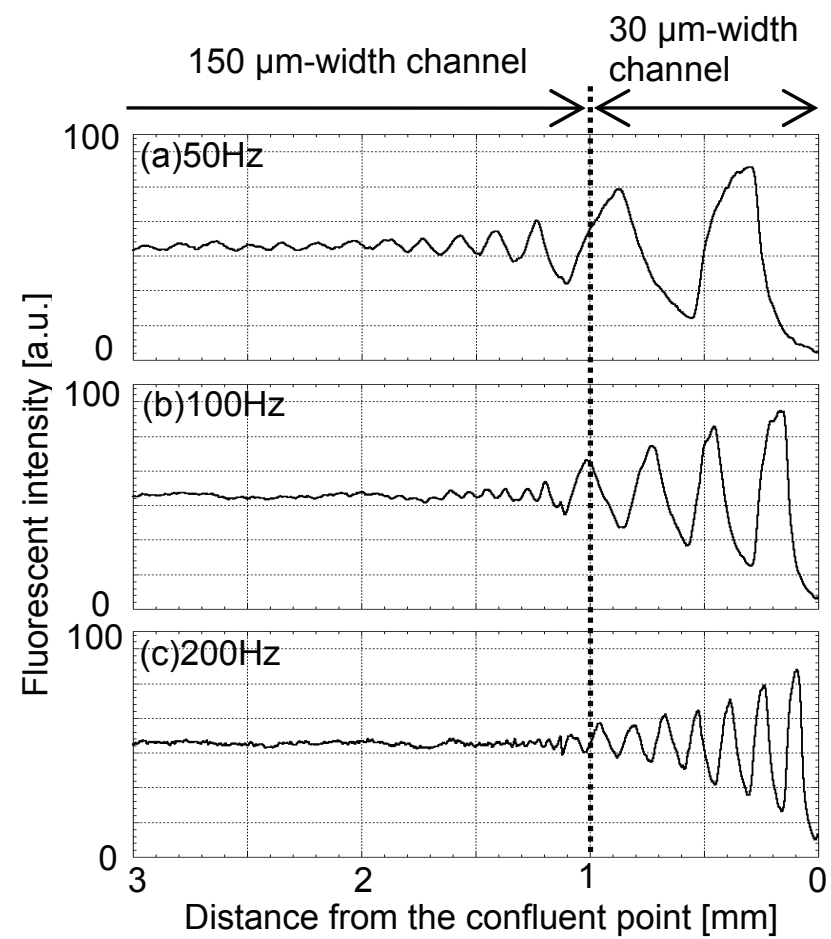

Fig. 7. Fluorescence intensity along the center of the microchannel at mid-depth for a switching frequency of a) $50 \mathrm{~Hz}$, b) $100 \mathrm{~Hz}$ and c) $200 \mathrm{~Hz}$. The fluorescence intensity values are relative values. 


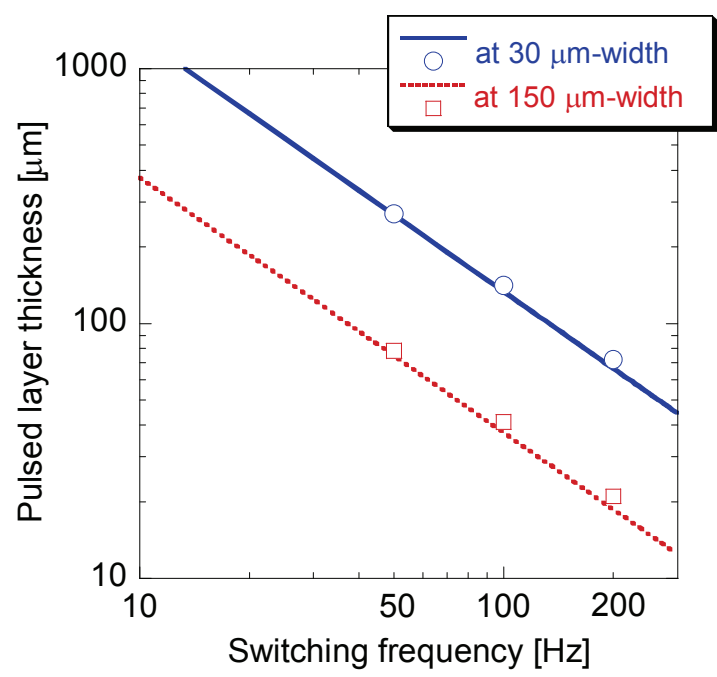

Fig. 8. Thickness of the pulsed layer as a function of switching frequency on a logarithmic scale. The solid and dotted line show the thickness of layers in the $30-\mu \mathrm{m}$ - and $150-\mu \mathrm{m}$-wide channels, respectively.

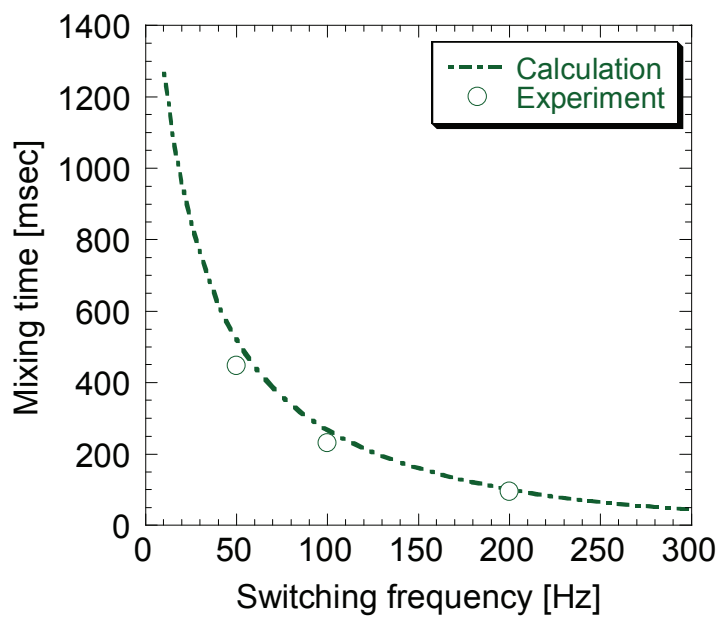

Fig. 9. Mixing time as a function of switching frequency. 


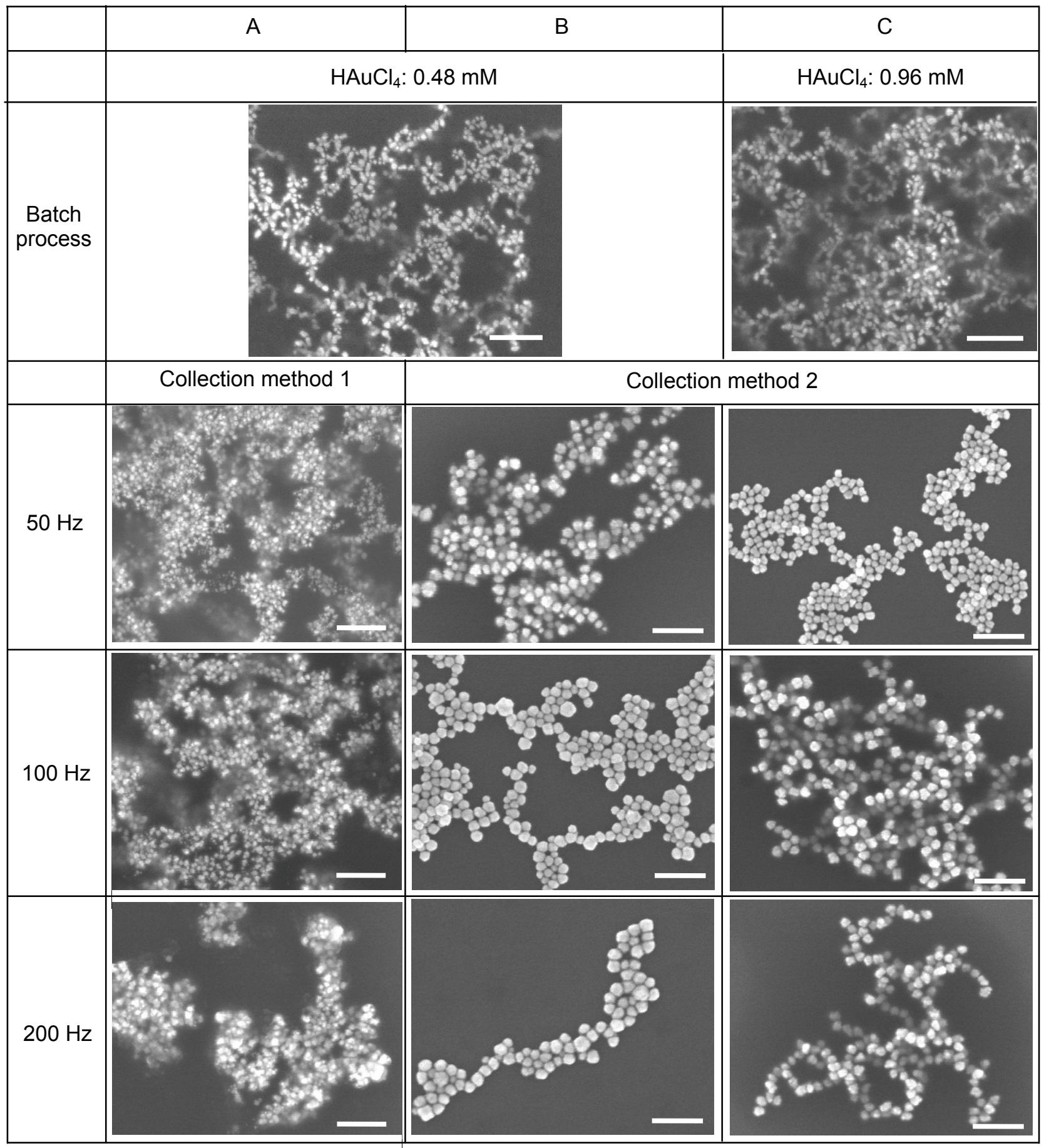

Fig. 10. SEM images of gold nanoparticles synthesized by batch process and pulsed mixing at switching frequencies of 50, 100 and $200 \mathrm{~Hz}$. Scale bars indicate $200 \mathrm{~nm}$. 
A

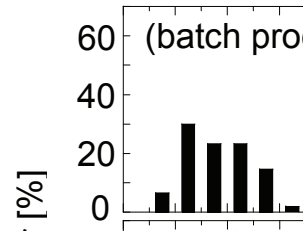

¿ 60 (a) $50 \mathrm{~Hz}$

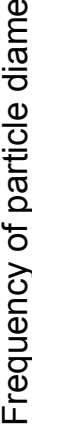

0 -(b) $100 \mathrm{~Hz}$

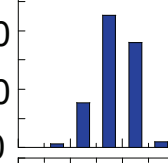

0 -(c) $200 \mathrm{~Hz}$
B

(a) $50 \mathrm{~Hz}$

(b) $100 \mathrm{~Hz}$

40

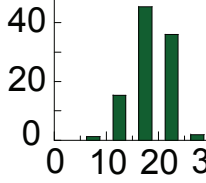

Particle diameter [nm]

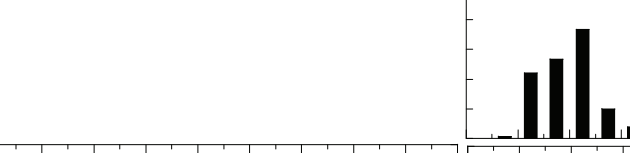

(a) $50 \mathrm{~Hz}$

(b) $100 \mathrm{~Hz}$

(c) $200 \mathrm{~Hz}$

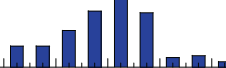

$\square$

(c) $200 \mathrm{~Hz}$

Fig. 11. Particle diameter distributions for gold nanoparticles synthesized by the batch process and the pulsed mixing method at switching frequencies of (a) $50 \mathrm{~Hz}$, (b) $100 \mathrm{~Hz}$ and (c) $200 \mathrm{~Hz}$. A) collection method 1 with a $\mathrm{HAuCl}_{4}$ concentration of $0.48 \mathrm{mM}$. B) collection method 2 with a $\mathrm{HAuCl}_{4}$ concentration of $0.48 \mathrm{mM}, \mathrm{C}$ ) collection method 2 with a $\mathrm{HAuCl}_{4}$ concentration of 0.96 $\mathrm{mM}$.

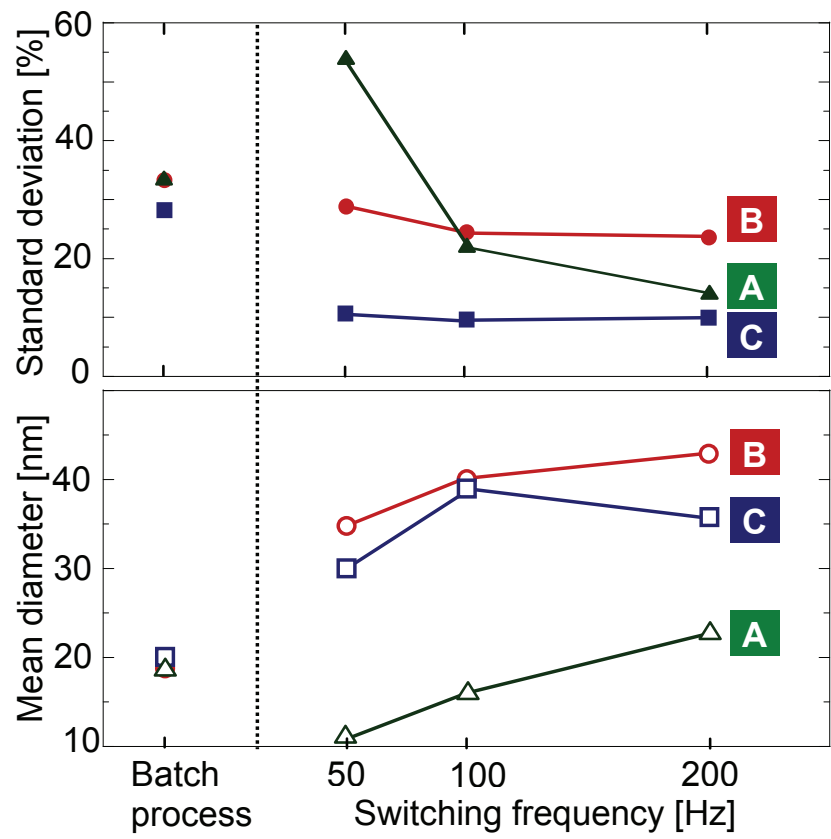

Fig. 12. Mean particle diameter and coefficient of variation for gold nanoparticles synthesized by the batch process and the pulsed mixing method at switching frequencies of (a) $50 \mathrm{~Hz}$, (b) $100 \mathrm{~Hz}$ and (c) $200 \mathrm{~Hz}$. (A) collection method 1 with a $\mathrm{HAuCl}_{4}$ concentration of $0.48 \mathrm{mM}$. (B) collection method 2 with a $\mathrm{HAuCl}_{4}$ concentration of $0.48 \mathrm{mM},(\mathrm{C})$ collection method 2 with a $\mathrm{HAuCl}_{4}$ concentration of $0.96 \mathrm{mM}$. 